\title{
Reviewing and Content Analysis of Persian Language Mobile Health Apps for COVID-19 Management
}

\author{
Leila ERFANNIA a,b, Morteza AMRAEI ${ }^{\mathrm{c}}$, Goli ARJI ${ }^{\mathrm{d}}$, Azita YAZDANI ${ }^{\mathrm{b}, 1}$, \\ Maryam SABZEHGAR ${ }^{\mathrm{a}}$ and Leila YAGHOOBI ${ }^{\mathrm{a}}$ \\ ${ }^{a}$ Health information technology department, Paramedical school, Zahedan University \\ of Medical Sciences, Zahedan, Iran \\ ${ }^{\mathrm{b}}$ Clinical education research center, health human resources research center, School \\ of health management and information sciences, Shiraz University of Medical \\ Sciences, Shiraz, Iran \\ ${ }^{c}$ Department of health information technology, faculty of allied medical sciences, \\ Lorestan university of medical sciences, Khorramabad, Iran \\ ${ }^{\mathrm{d}}$ Saveh university of medical sciences, school of nursing and midwifery, health \\ information technology department, Saveh, Iran
}

\begin{abstract}
The present study aimed to systematically search in app stores and intended to carry out content analysis of free Persian mobile health apps in the management of COVID-19 and, ultimately determine the relationship between the popularity and quality of these apps. According to a researcher-made checklist including five axes of ease of use, privacy, data sharing, education, and monitoring, four app markets such as Myket, Bazzar, Google Play and App Store were searched from May 2021 up to now. The findings showed that all selected apps performed well in terms of ease of use and privacy but they needed to be improved in terms of education, monitoring, and data sharing. Also, there was no significant relationship between the popularity and quality of these apps. Owing to the high penetration rate of smartphones in Iran and the low popularity of COVID-19 apps, government, developers, and investors are required to improve the quality of apps and their marketing.
\end{abstract}

Keywords: Mobile health app, COVID-19, Persian app, Content analysis.

\section{Introduction}

Recently, outbreak of SARS-CoV-2 pandemic and the subsequent increase in mortality rate stressed the need for gaining information on various aspects of the virus [1]. Of course, The number of mobile health (m-health) apps has grown dramatically since the outbreak of the COVID-19 pandemic. COVID- related-m-health apps enjoy a number of other useful functions, including contact tracing and symptom control by health care

\footnotetext{
1 Corresponding author, Azita YAZDANI Ph.D. of Medical Informatics, Assistant Professor, Clinical Education Research Center, Health Human Resources Research Center, School of Health Management and Information Sciences, Shiraz University of Medical Sciences, Shiraz, Iran. Email: a_yazdani@sums.ac.ir. ORCID ID: 0000-0002-5190-286X
} 
professionals and monitoring the number of infections [2].Hence, Evaluating the content and features of mobile apps is very important to guide users in choosing the right mobile app based on their needs. It should be noted that the studies conducted so far to evaluate the quality of COVID-related apps are mainly in English [3]. Also, A study that focused on Persian language considered all apps and systems and evaluated them based on $\boldsymbol{M A R S}$ scale [4].Accordingly, the present study focuses solely on free Persian m-health apps in managing COVID-19 and uses a researcher-made checklist based on specific pandemic features to evaluate the apps.

\section{Method}

Based on PRISMA 2020 steps, to find Persian mobile apps, Myket, App Store and Bazaar mobile app stores were selected due to the coverage of Persian $m-H e a l t h$ apps and App Store and Google Play platforms and web search were also selected to get the desired results. Then, Persian keywords including "كرونا" and "19-19 from May 2021 were considered as our main search. Inclusion Criteria, here, included mobile apps that launched at the time of the epidemic, and focused on the management of COVID disease in terms of diagnosis, education, follow-up and counseling. 46apps selected for full review. At this stage, the assessors fully evaluated the remaining apps regarding capabilities and functions and excluded apps that focused only on one aspect and did not cover other related areas. Then 21 apps entered the final evaluation stage (Figure1).

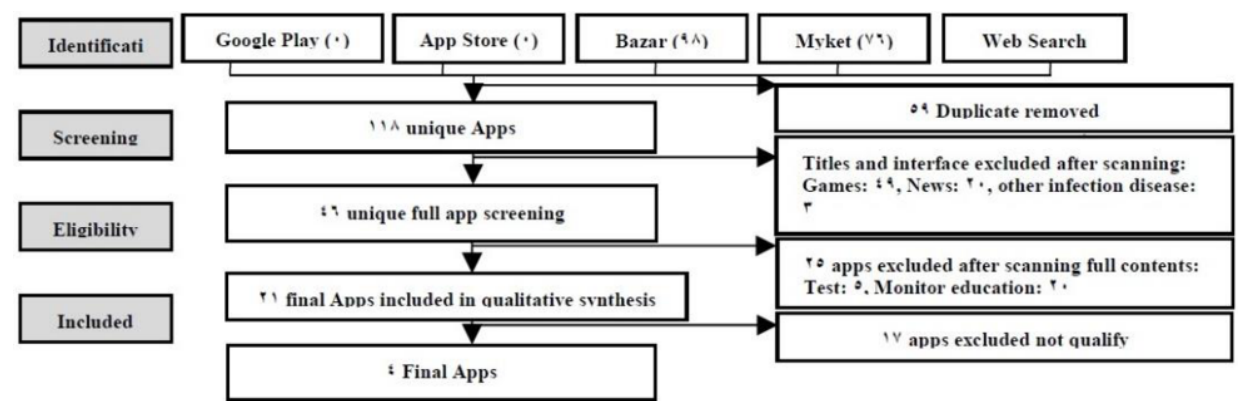

Figure1. The PRISMA flowchart of the screening process of the reviewed national Persian apps related to COVID

A researcher-made checklist (this is available upon request from the corresponding author) was designed based on $\mathrm{m}$-Health programs evaluation criteria, literature review, and WHO guidelines on digital health interventions to classify the app functions [5-7]. It consisted of 37 questions in five axes (includes: ease of use, privacy, data sharing, education and monitoring) scored on a two-point scale, one for Yes and zero for No. Two assessors evaluated each app independently and every discrepancy was dealt with in a joint meeting with a third assessor. Subsequently, Apps with scores higher than the average score (18.5) were rated as acceptable. Accordingly, only did four apps receive acceptable scores, and the others were excluded from the study. 


\section{Results}

All mobile apps in the qualitative step were evaluated according to each of the five axes. Five items were examined in ease of use axis namely as: flexibility and customization, understandable figures and icons, application assistance, data entry, and page forward and backward easiness). Overall, Mask, AC19 and Safirane Salamat apps got the most points from this axis ( 5 points each). Four items were examined in privacy axis -i-eLegal commitment, username and password, username and passwords change, reregistration). Safirane Salamat and Corona (online diagnosis/statistics/education) apps obtained the highest scores in this axis (4 points each). Eleventh items were reviewed in data sharing axis-i-e- access to health history, volunteers assistance in crisis, search in app, send health reminders, link to educational pages, demographic data entry, data sharing from app, automatic data entry from user profile, online consultations, users feedback entry, users experience sharing and forums ). The highest score in this axis belonged to Safirane Salamat, Corona (online diagnosis/statistics/education), and AC19 apps (9, 6, 6 points respectively). In education axis, nine items were examined as follows: education by images, health events information, patients and relatives educations, COVID death information, latest COVID cases according geographical parts, COVID statistics by tables and graphs, prediction COVID pattern in future, provide prevention video, send periodic massages for users ). Overall, AC19 app obtained the highest score in this axis (Score 9 out of 9). In monitoring axis, eight items were examined as follows: user tracking, patients monitoring from home, introducing labs and medical imaging centers, follow up patient's symptoms, evaluating possibility of COVID infected, monitored quarantine, introducing symptoms which need physician visit, providing health center address and map). Altogether, Safirane Salamat and Mask apps received the highest scores in this axis (6 points each). Having determined the final score of the apps, the download rate was extracted based on the download statistics of the app stores. Then, Pearson correlation test was used to test the relationship between the total score of the apps and the download rate and, of course, no significant correlation was observed between them $(\mathrm{p}=0.690, \mathrm{r}=0.310)$.

Table1. Final national Persian COVID-19 mobile apps features.

\begin{tabular}{llllll}
\hline App Name & $\begin{array}{l}\text { Star } \\
\text { rating/form }\end{array}$ & Download & Main Features & Platform & $\begin{array}{l}\text { Size } \\
\text { (Meg) }\end{array}$ \\
\hline Safirane Salamati & $4 / 169$ & +23000 & Education & Android/iOS & 25.3 \\
\hline AC19 & 4.1 & +50000 & $\begin{array}{l}\text { Risk } \\
\text { assessment/self- } \\
\text { assessment }\end{array}$ & Android & 5 \\
& $/ 6651$ & & $\begin{array}{l}\text { Education/risk } \\
\text { assessment }\end{array}$ & Android & 27 \\
\hline Mask & 4.1 & +700000 & $\begin{array}{l}\text { Education/ risk } \\
\text { assessment }\end{array}$ & Android & 14 \\
\hline $\begin{array}{l}\text { Corona(online } \\
\text { diagnosis/statistics/education) }\end{array}$ & $3.5 / 11$ & +500 & & & \\
\hline
\end{tabular}

Based on the total points, the Safirane Salamat app obtained the highest score (score of 29), followed by Ac19 (score of 26) and Mask (score of 21). These selected apps, and Corona (online diagnosis/statistics/education); (score of 19), obtained more than $50 \%$ of the quality review score. The features of final selected apps are demonstrated in Table1. 


\section{Discussion}

Needless to say that Public-private partnerships can contribute to the applications success and involve in achieving health goals [8]. Mask app, the most popular Persian m-health app, was launched through the government/ non- government participation. Reportedly, the ease of using m-health apps and education seems to be one of the effective items for m-health apps. All four apps received acceptable scores for ease of use. User education is one of the capabilities of $\mathrm{m}$-health apps in preventing the spread of disease. AC19 app obtained the highest scores in this area and provided education content. The only Persian app which was capable of tracking users' calls from the beginning was the Mask. Mask and Safirane Salamat were able to provide strategies to support people in quarantine. According to the results of the correlation analysis, there was no significant relationship between the popularity of apps and their quality, which is consistent with the results of Wang's study [9].Hence, it can be concluded that the popularity of apps such as ads or search engine optimizations can be increased without necessarily being a higher quality than other apps. The final apps have been successful in ease of use and privacy. These apps need to be upgraded in terms of data sharing, education, and monitoring. Online consultation were not considered in the selected apps, though. As far as education axis was concerned, most apps provided educational information from the Ministry of Health and Official Scientific resources due to government affiliation, which were the strong points of these apps. In terms of monitoring, apps were only capable to assess the risk and make recommendations to visit the doctor, and were not able to monitor patients or suspected cases or people in quarantine. User tracing and quarantine monitoring were among the most important features of COVID- 19 apps, which were neglected in Persian $\mathrm{m}$-health apps, though. The results of the present study showed that the most successful Persian m-health apps have been designed, launched, and even marketed through government support.

\section{References}

[1] Abd-Alrazaq A, et al. Top concerns of tweeters during the COVID-19 pandemic: infoveillance study. Journal of medical Internet research, 2020 March;22(4):e19016.

[2] Abeler J, et al. COVID-19 contact tracing and data protection can go together. JMIR mHealth and uHealth, 2020 April;8(4):e19359.

[3] Blacklow SO, et al. Usability, inclusivity, and content evaluation of COVID-19 contact tracing apps in the United States. Journal of the American Medical Informatics Association, 2021 June;28(9):1982-9.

[4] Abdolkarimi S, Mousavi B. Content Analysis of Health-Based Iranian Systems and Apps on Covid-19. Iranian Journal of War and Public Health, 2021 March;12(4):223-233.

[5] Ming LC, et al. Mobile health apps on COVID-19 launched in the early days of the pandemic: content analysis and review. JMIR mHealth and uHealth, 2020 Sep;8(9):e19796.

[6] Nouri R, et al. Criteria for assessing the quality of mHealth apps: a systematic review. Journal of the American Medical Informatics Association, 2018 Aug;25(8):1089-98.

[7] Guideline W. Recommendations on digital interventions for health system strengthening. World Health Organization, 2019:2020-10.

[8] Zhou X, Snoswell CL, Harding LE, Bambling M, Edirippulige S, Bai X, Smith AC. The role of telehealth in reducing the mental health burden from COVID19. Telemedicine and e-Health. 2020;26(4):377-9

[9] Wang X, Markert C, Sasangohar F. Investigating Popular Mental Health Mobile Application Downloads and Activity During the COVID-19 Pandemic. Human Factors. 2021 Mar;0018720821998110. 